\title{
Innovación empresarial al servicio de la micro y pequeña empresa nortesantandereana: por la competitividad regional ${ }^{1}$
}

\section{Business innovation at the service of the micro and small business of North-Santander: for regional competitiveness}

DOI: https://doi.org/10.17981/econcuc.40.1.2019.06

Artículo de investigación. Fecha de recepción: 23/08/2018 Fecha de aceptación: 15/03/2019

\author{
Claudia Milena Pérez \\ Corporación Universitaria Minuto de Dios. (Cúcuta, Colombia) \\ cperez3@uniminuto.edu.co
}

Para citar este artículo:

Pérez, C. (2019). Innovación empresarial al servicio de la micro y pequeña empresa nortesantandereana: por la competitividad regional. Económicas CUC, 40(1). 91-104. DOI: http://doi.org/10.17981/econcuc.40.1.2019.06

\section{Resumen}

La innovación empresarial es una tendencia indispensable para la competitividad en medio de crecientes exigencias del mercado en materia de oportunidades y valor agregado de servicios y productos. La investigación tuvo por objeto formular una herramienta para medir la capacidad innovadora de la empresa nortesantandereana, a través de una revisión de fuentes conceptuales de innovación empresarial, y una encuesta dirigida en 50 empresas, cuyos resultados sumados a los referentes teóricos permitieron operacionalizar variables de éxito medibles para evaluar la capacidad de innovación (CI) empresarial, basándose en la propuesta de la Organización para la Cooperación y el Desarrollo Económicos-OCDE (Manual de Oslo), desde las perspectivas técnica, organizativa y administrativa. Se concluye que la capacidad de innovación empresarial implica una administración efectiva de los elementos clave.

Palabras clave: Innovación empresarial; capacidad de innovación; competitividad regional y mundial; empresas nortesantandereanas.

\begin{abstract}
Entrepreneurial innovation is an indispensable trend for competitiveness amid growing market demands for opportunities and added value of services and products. The purpose of the research was to formulate a tool to measure the innovating capacity of the Nortesantandereana company, through a review of conceptual sources of business innovation, and a survey conducted in 50 companies, whose results, added to the theoretical references, made it possible to operationalize measurable success variables to evaluate the innovation capacity (CI) of the company, based on the proposal of the Organization for Economic Cooperation and Development-OECD (Oslo Manual), from the technical, organizational and administrative perspectives. It is concluded that the capacity for business innovation implies an effective administration of the key elements.
\end{abstract}

Keywords: Business innovation; innovation capacity; regional and global competitiveness; Nortesantanderean companies.

\footnotetext{
${ }^{1}$ Artículo de investigación científica y tecnológica, resultado del proyecto de investigación "Diseño y validación de herramienta de medición de innovación, al servicio de la micro y pequeña empresa Nortesantandereana” desarrollado por la línea de investigación innovaciones sociales y productivas del Grupo de investigación en Desarrollo Humano, Tejido social e Innovaciones Tecnológicas GIDTI, Semillero Reinnova, Corporación Universitaria Minuto de Dios - CR Cúcuta..
} 


\section{INTRODUCCIÓN}

Norte de Santander es un departamento colombiano fronterizo con Venezuela, cuya economía se basa principalmente en el comercio de bienes y servicios (Gobernación de Norte de Santander, 2016), y recientemente se encuentra afectado por la ruptura de las dinámicas económicas binacionales derivadas del cierre fronterizo decretado por el país vecino desde 2015. Esta realidad ha exhortado a los empresarios de la región a asumir nuevos retos para fortalecer la economía de la región, mediante la búsqueda de nuevas posibilidades de desarrollo socioeconómico a través del sector industrial, considerando que las micro y pymes (Mipymes) son un componente fundamental del tejido empresarial en América Latina (Dini, Rovira \& Stumpo, 2014), mismas que han asumido a parte de los trabajadores desplazados como consecuencia del cierre fronterizo mencionado y que han generado como producto una nueva versión en el campo empresarial (Pérez, Hernández, Acosta y Chumaceiro, 2009), para fortalecer la competitividad y ampliar el acceso a nuevos mercados nacionales, internacionales e incluso el local.

Desde los años noventa, muchos países adoptaron la gestión de la innovación en las empresas para promover la competitividad empresarial; OCDE (2006) afirma que "la innovación en la empresa se refiere a los cambios previstos en sus actividades y que están orientados a mejorar sus resultados" (p. 43), mientras que Ochoa (2007) citado por González (2015) formula que "la innovación es un proceso sistémico donde convergen distintas disciplinas del conocimiento, de una o varias empresas, con el propósito de explorar y encontrar mejoras a los mercados, productos o procesos, devolviendo un beneficio económico y ambiental a la empresa y la sociedad" (p. 8).
Dada la dinámica regional, los empresarios nortesantandereanos asumirían la gestión de la innovación empresarial desde el interés y los riesgos calculados para aplicar cambios internos significativos en el que hacer organizacional, tal y como lo demandan la globalización y las nuevas exigencias de los mercados, cada vez más individualizados y marcados en la diferencia; un primer paso para materializar dicha gestión es conocer el estado de la empresa, desde la identificación de sus fortalezas y debilidades para desarrollar planes de fortalecimiento.

La generalización de las encuestas globales de innovación desde hace más de 15 años, generó una proliferación de estudios relativos a las condiciones determinantes de la innovación y su impacto en el desempeño de las empresas. Un resultado clave y sistemático en la mayor parte de dichos estudios indica que las diferencias en capacidades, conexiones y tamaño afectan significativamente la conducta innovadora de las empresas; en general, los resultados develan que las empresas con mayor capacidad de absorción y conectividad con otros agentes y más grandes, tienen mayores posibilidades de innovar (Comisión Económica para América Latina y el Caribe-CEPAL, 2010. p. 25).

Conocedores de esta realidad, el Semillero REINNOVA del programa de Administración de empresas y administración financiera adelantó en el año 2016-2017 la presente investigación, para diseñar y validar una herramienta de medición de las capacidades innovadoras de la empresa nortesantandereana, partiendo de las capacidades empresariales como el estudio de "los elementos que le permiten sacar partido de las oportunidades ofrecidas por el mercado" (OCDE, 2006, p. 162), 
desde la identificación de los elementos organizativos, técnicos y administrativos mínimos que se requieren para gestionar la innovación, mediante un diagnostico interno de su estado en pro del aprovechamiento efectivo de los recursos empresariales y las oportunidades del mercado.

La investigación consideró la línea base de necesidades empresariales para fortalecer las capacidades de innovación, las acciones planificadas y oportunas de acompañamiento y la formación ofrecida por la Corporación UniMinuto Cúcuta, a las micro y pequeñas empresas regionales con interés y requerimientos de apoyo, a las que por razones económicas o desconocimiento no pueden acceder para mejorar su competitividad.

\section{Metodología}

Esta investigación descriptiva se fundamentó en una revisión sistemática de contenidos y un trabajo de campo empresarial adelantados en tres fases: sistematización de información y variables a partir de las fuentes secundarias y encuentros con empresarios; análisis de datos, desarrollo y validación de la herramienta de caracterización empresarial; y plan de divulgación de los resultados.

Fase 1. Recolección de información secundaria: Se fundamentó en la revisión documental para determinar la variable y los indicadores pertinentes de medición de la innovación empresarial bajo el enfoque investigativo formulado; se complementó con encuentros de empresarios para identificar los rasgos, variables e indicadores culturales que eventualmente inciden en la innovación empresarial.

Fase 2. Análisis de datos y elaboración de herramienta: Mediante la operacionalización de variables para medir la innovación empresarial se desarrolló el instrumento encuesta para medir la capacidad de innovación empresarial, basada en la formulación de premisas y la medición bajo escala Likert. Se diseñó un programa informático para tabular y analizar los resultados de dichas mediciones, y se aplicó la encuesta en modalidad de validación de diseño y contenidos en 50 empresas de sectores representativos del departamento.

Fase 3. Presentación de resultados: Se desarrolló un informe diagnóstico individual de innovación empresarial para cada empresa participante, y un consolidado de tendencias conformado por puntos de acuerdo entre los participantes a partir del cual se elaboró el informe final; para socializar los resultados se convocaron empresarios o sus representantes con quienes se presentaron y validaron los hallazgos principales de la investigación, la que a su vez fue postulada para publicación en medio de divulgación indexado.

\section{Diseño de instrumentos: Muestra y recolección de datos}

Se desarrollaron dos instrumentos para la recolección, tabulación y análisis de información de campo, cuyo fin fue identificar las debilidades y fortalezas en materia de capacidad innovadora de las empresas participantes en la investigación.

\section{1) Instrumento de recolección de información}

Consistió en la aplicación de una entrevista dirigida a los propietarios, gerentes o delegados de las empresas participantes del estudio, cuyas preguntas hicieron referencia a la operacionalización de las variables relacionadas en la tabla 1 , orientadas en el ciclo de gestión de Deming de la mejora continua, PHVA (planear, hacer, 
verificar y actuar); este ejercicio proveyó información básica, técnica y organizativa de la empresa para medir sus capacidades de innovación en el quehacer empresarial.

TABLA 1

Variables teóricas que caracterizan la capacidad de innovación empresarial.

\begin{tabular}{|c|c|c|}
\hline $\begin{array}{l}\text { Variable } \\
\text { teórica }\end{array}$ & Referentes teóricos & Subvariables \\
\hline 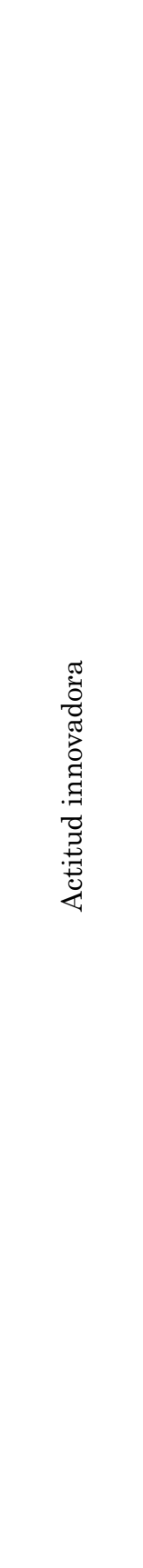 & $\begin{array}{l}\text { "La actitud de la empresa } \\
\text { ante la innovación indica } \\
\text { la postura de una empresa } \\
\text { hacia el liderazgo y } \\
\text { vanguardia tecnológica en } \\
\text { su sector de actividad"; la } \\
\text { actitud empresarial con } \\
\text { liderazgo de los propietarios, } \\
\text { directivos y trabajadores, } \\
\text { afronta mejor los cambios } \\
\text { y retos y aprovecha los } \\
\text { avances tecnológicos para } \\
\text { mejorar su actividad } \\
\text { empresarial (Porter, Zahra } \\
\text { \& Covin, citado por Donate } \\
\text { y Guadamillas, 2010, p. } \\
\text { 34). } \\
\text { El liderazgo innovador se } \\
\text { fortalece con conocimiento, } \\
\text { experiencia, cambios, } \\
\text { riesgos y para generar } \\
\text { un valor diferenciador, } \\
\text { relacionamiento con el } \\
\text { entorno y en los bienes o } \\
\text { servicios ofertados; también } \\
\text { cuenta el "conocimiento de } \\
\text { las empresas, las aptitudes } \\
\text { de los trabajadores, su } \\
\text { formación educativa, } \\
\text { utilización de las TIC, } \\
\text { proximidad de instituciones } \\
\text { públicas de investigación } \\
\text { y regiones con densidad } \\
\text { innovadora" (OCDE, 2006, } \\
\text { p. 104). } \\
\text { Según Avendaño (2012), } \\
\text { la innovación se origina } \\
\text { en el desarrollo de nuevas } \\
\text { ideas, que sumadas al valor } \\
\text { marcan una diferencia con } \\
\text { la creatividad como mera } \\
\text { capacidad; la innovación } \\
\text { requiere gestión del } \\
\text { conocimiento para lograr } \\
\text { sus objetivos y conquistar el } \\
\text { mercado (p. 189). }\end{array}$ & $\begin{array}{l}\text { Promoción de la cultura de la innovación: parte } \\
\text { del liderazgo asumido por propietarios y directivos para } \\
\text { gestionar nuevas ideas, motivar la creatividad y la gestión } \\
\text { empresarial ante los cambios requeridos. Implica la } \\
\text { actitud adecuada de los trabajadores frente a los cambios } \\
\text { empresariales innovadores mediante la formación integral } \\
\text { y permanente, los incentivos y la motivación para la } \\
\text { creatividad empresarial. Bes y Kotler (2011) advierten que } \\
\text { si nadie se ocupa de activar estas dinámicas, se corre el } \\
\text { riesgo de permanecer en la rutina (p. 35). } \\
\text { Recursos internos para innovar: Además de apropiar } \\
\text { la cultura de la innovación en el quehacer empresarial, } \\
\text { la gestión innovadora demanda recursos económicos, } \\
\text { logísticos, humanos e infraestructura para materializar } \\
\text { los cambios; los principales recursos son de financiamiento } \\
\text { de acciones definidas y el uso asertivo de los sistemas } \\
\text { de información y comunicación (TIC) para la innovación } \\
\text { empresarial. }\end{array}$ \\
\hline
\end{tabular}


$\cdots$

$\begin{array}{lll}\begin{array}{c}\text { Variable } \\ \text { teórica }\end{array} & \text { Referentes teóricos } & \text { Subvariables }\end{array}$

Actividades innovadoras de producto: Según la OCDE (2006) corresponde a introducir un bien o servicio nuevo o significativamente mejorado (p. 58), dada la exigencia de los mercados en función de los avances tecnológicos, y la necesidad de atender requerimientos específicos de los

La capacidad innovadora empresarial involucra, actuaciones científicas, tecnológicas, organizativas, financieras y comerciales que innovan en sí mismas, mientras que otras pueden sin hacer esto, ser necesarias para introducir otras innovaciones (OCDE, 2006, p. 25).

La innovación involucra actividades no incluidas en I+D de preproducción, producción y distribución, como el apoyo formativo $\mathrm{y}$ preparación del mercado para innovar productos, desarrollar y aplicar nuevos métodos de comercialización y organización (OCDE, 2006, p.104); las empresas innovan para mejorar sus resultados, aumentando la demanda o reduciendo costos.

La OCDE (2006) define cuatro actividades innovadoras a saber: $d e$ producto, de proceso, de mercadotecnia y de organización; según estas, el aumento de la demanda obedece a la innovación de producto y mercadeo, y la reducción de costos depende de la innovación de proceso y organización, para lograr ganancias de productividad y un modelo de interacción y generación de valor para optimizar recursos y aumentar la rentabilidad. clientes mediante soluciones personalizadas y mejoras del producto. La traducción creativa del conocimiento y análisis del mercado genera productos nuevos o con valor, evaluables en función de su aceptación, rentabilidad y relaciones redituables con los clientes, para determinar el posicionamiento empresarial innovador en el mercado y en su propio sector. Actividades innovadoras de proceso: Tienen por objeto agregar valor en la cadena productiva de la empresa, adquiriendo o mejorando los materiales, técnicas, maquinarias, equipos y herramientas productivas, y adquiriendo o mejorando el conocimiento técnico; considera los medios logísticos de distribución, programas informáticos, técnicas de abastecimiento de insumos, suministros y distribución de productos finales (OCDE, 2006 , p. 59). Innovar en proceso, "busca especialmente los mejoramientos que redunden en la reducción de tiempos y por lo tanto de costos operativos" (Ángel, 2005).

Actividades innovadoras de mercadeo: Buscan satisfacer las necesidades de los consumidores, abrir nuevos mercados o posicionar productos para aumentar las ventas (OCDE, 2006, p. 60), mediante la implementación de nuevos métodos de mercadeo y comercialización basados en: la identificación de requerimientos de los clientes actuales y potenciales mediante el relacionamiento con ellos; el estudio de tendencias y dinámicas nacionales y mundiales; al acondicionamiento del producto y envase; la generación de estrategias de tarificación de precios; la implementación de nuevos canales de venta; el uso de estrategias de publicidad y promoción asertivas; y la implementación de métodos novedosos de comercialización. El estudio y planeación de mercados y tendencias mundiales, permite que la empresa tome decisiones para desarrollar y mejorar sus procesos y productos, y responder al mercado cautivo y los nuevos mercados. Actividades innovadoras de organización: Son los nuevos cambios y métodos organizativos en las prácticas, la organización del lugar de trabajo o las relaciones exteriores de la empresa (OCDE, 2006), derivados de la planeación estratégica, cuyo fin es interconectar recursos y áreas funcionales para definir un modelo de negocios producto y destacado entre su competencia. El modelo de negocio describe las forma en que la empresa crea, proporciona y capta valor" (Osterwalder y Pigneur, 2011, p. 14).El valor no reposa en el producto o servicio, pues de ser así, no representa una ventaja competitiva en tanto que la competencia tiene acceso a él. La oportunidad de valor organizacional debe integrar las competencias de sus unidades, áreas o departamentos, y trabajar sistemáticamente con nuevos procesos (Chiavenato, 2011, p. 83). 


\begin{tabular}{|c|c|c|}
\hline $\begin{array}{l}\text { Variable } \\
\text { teórica }\end{array}$ & Referentes teóricos & Subvariables \\
\hline 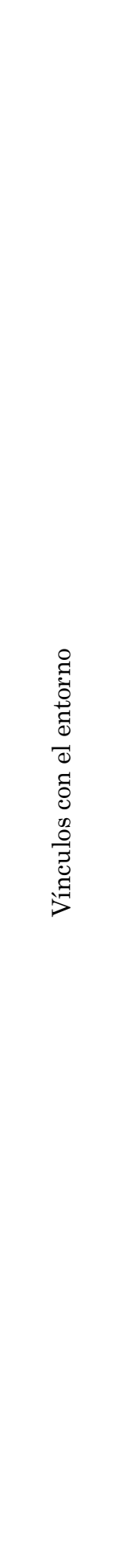 & 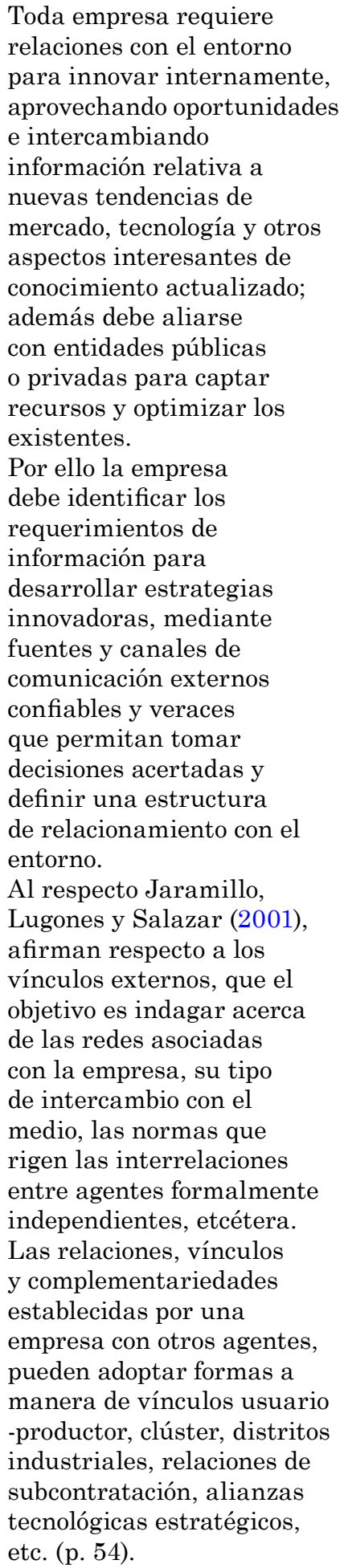 & $\begin{array}{l}\text { Información para la innovación: Los vínculos inter } \\
\text { empresariales obedecen al acceso, tipo de información } \\
\text { que intercambian, o articulación lograda. Puede tenerse } \\
\text { información gratuita de libre acceso o información científica } \\
\text { - tecnológica con costos, adquirida mediante compra } \\
\text { directa o cooperación con la fuente mediante experiencias } \\
\text { y asesorías mediadas por derechos y patentes. En este } \\
\text { sentido, la empresa debe contar con un responsable de } \\
\text { la gestión de recursos para adquirir dicha información } \\
\text { potencialmente aprovechable en la organización. } \\
\text { Relacionamiento con el entorno para la innovación: } \\
\text { Otros vínculos se derivan de la participación activa de } \\
\text { la empresa con organizaciones estatales o privadas para } \\
\text { desarrollar acciones conjuntas para gestionar proyectos y } \\
\text { desarrollar objetivos que representen beneficios recíprocos. } \\
\text { (OCDE, 2006, p. 92). } \\
\text { El trabajo colectivo favorece y optimizar los recursos en } \\
\text { innovación y desarrollo competitivo de las micro y pequeñas } \\
\text { empresas, en tanto su sumatoria de intereses, objetivos } \\
\text { y medios, genera cambios de mayor impacto para la } \\
\text { implementación de acciones innovadoras que favorezcan su } \\
\text { sostenibilidad y crecimiento colaborativo. }\end{array}$ \\
\hline
\end{tabular}

Fuente: Elaboración propia mediante adaptación de varios autores. 
2) Instrumento de tabulación y análisis gráfico de información

$A$ partir de la información de campo se desarrolló un programa informático para asignar un valor cuantitativo a las variables de acuerdo con los criterios definidos en el semillero; la valoración se realizó considerando un peso relativo del $100 \%$ para cada categoría y distribuyéndolo entre las subcategorías, los indicadores y las micro variables en forma proporcional y ponderada de acuerdo con su representatividad. A su vez el peso se contrarrestó con la valoración de las premisas de éxito de las capacidades de innovación en función de la escala de Likert y la información recolectada:

TABLA 2

Criterios de valoración cuantitativa de indicadores

de medición de capacidad innovadora en la empresa

\begin{tabular}{|c|c|c|c|}
\hline Cuantitativo & $\begin{array}{l}\text { Valor } \\
\text { Cualitativo - nominal } \\
\text { (Escala de Likert) }\end{array}$ & \multicolumn{2}{|c|}{$\begin{array}{l}\text { Criterios para evaluar los componentes } \\
\text { de capacidad de innovación } n^{*}\end{array}$} \\
\hline 1 & $\begin{array}{l}\text { Totalmente en } \\
\text { desacuerdo }\end{array}$ & \multirow{5}{*}{ La empresa: } & $\begin{array}{l}\text { No considera importante } \\
\text { el componente ni está } \\
\text { disponible para su trabajo. }\end{array}$ \\
\hline 2 & En desacuerdo & & $\begin{array}{l}\text { Desconoce el componente } \\
\text { y su importancia para la } \\
\text { promoción de la innovación } \\
\text { empresarial; mas tiene } \\
\text { interés en su práctica inicial. }\end{array}$ \\
\hline 3 & $\begin{array}{l}\text { Ni de acuerdo ni en } \\
\text { desacuerdo }\end{array}$ & & $\begin{array}{l}\text { Conoce el componente, } \\
\text { ha realizado algunas } \\
\text { prácticas con este como } \\
\text { acciones aisladas (no } \\
\text { planificadas), pero reconoce } \\
\text { su importancia y se interesa } \\
\text { por mejorar su práctica. }\end{array}$ \\
\hline 4 & De acuerdo & & $\begin{array}{l}\text { Conoce el componente, } \\
\text { ha realizado algunas } \\
\text { prácticas con este como } \\
\text { acciones planificadas, y está } \\
\text { organizando los recursos } \\
\text { para promocionar la } \\
\text { innovación empresarial. }\end{array}$ \\
\hline 5 & Totalmente de acuerdo & & $\begin{array}{l}\text { Conoce el componente, } \\
\text { planea, organiza y dirige } \\
\text { su implementación, y } \\
\text { ejecuta controles y mejoras } \\
\text { continuas para fortalecer la } \\
\text { innovación empresarial. }\end{array}$ \\
\hline
\end{tabular}

* Los criterios de evaluación fueron establecidos de acuerdo con los resultados de la entrevista dirigida de diagnóstico de capacidades aplicada a las empresas

Fuente: Elaboración propia. 
Para validar el instrumento se priorizaron empresas nortesantandereanas representativas de los sectores agropecuario, agroindustrial, industrial y de servicios, mientras que la aplicación del mismo se seleccionaron 50 de ellas que fueron abordadas por 18 estudiantes de los semilleros de investigación del programa de administración de empresas y financiera de la UniMinuto CR Cúcuta; la distribución de las empresas seleccionadas en la muestra se realizó de la siguiente forma:

TABLA 3

Tipo y cantidad de empresas participantes

\begin{tabular}{cc}
\hline \multicolumn{2}{c}{ Sector primario } \\
\hline Agrícola & 8 \\
Pecuario & 1 \\
Agropecuario & 3 \\
Minería & 3 \\
Subtotal & 15 \\
\hline Sector secundario & \\
\hline Agroindustria & 7 \\
Industrial & 20 \\
Subtotal & 27 \\
\hline Sector terciario & \\
\hline Servicios & 3 \\
Alimentos & 5 \\
Subtotal & 8 \\
\hline TOTAL & 50 \\
\hline
\end{tabular}

Fuente: Elaboración propia.

\section{Resultados}

Los resultados fundamentales de la investigación se concentraron en la construcción teórica de las variables de éxito de carácter medible, técnico, organizativo y administrativo, requeridas para evaluar la capacidad de innovación (CI) de las empresas nortesantandereanas en su quehacer empresarial, basándose en fundamentos epistemológicos de la propuesta de la OCDE (2006) conocida en las ciencias administrativas y afines como Manual de Oslo, y soportado por las teorías de Chiavenato (2011), Porter (2001), Kotler, Armstrong \& Benassini (2011) y Avendaño (2012), entre otros.

Según OCDE (2006), la CI de una empresa está definida por los "elementos que le permiten sacar partido de las oportunidades ofrecidas por el mercado" (p. 162), es decir, los elementos internos de la empresa y los de relacionamiento con otras organizaciones e instituciones que aporten a su mejoramiento productivo y la generación de ventajas competitivas para satisfacer necesidades, cumpliendo con las expectativas del mercado y afrontando retos y cambios del entorno en general.

Para lograr la CI es fundamental el conocimiento acumulado de la empresa incorporado en los recursos humanos, los procedimientos, procesos, capacidades tecnológicas y otros componentes, pues la curva de aprendizaje consciente y deliberado es costosa en tiempo y dinero, no lineal y dependiente de la trayectoria sostenida y acumulativa (OCDE, 2006, p. 162).

En resumen, la medición de la CI debe considerar los recursos humanos, los vínculos de la empresa y las tecnologías de la información y la comunicación TIC; a partir de dicha lógica, la medición de la CI de las empresas parte delas siguientes variables teóricas: 


\section{Operacionalización de variables para medir la $C I$}

El marco teórico analizado permitió operacionalizar las variables orientadoras de la medición de la CI empresarial, bajo un análisis realizado a través de la matriz conformada por tres (3) categorías teóricas derivada en subcategorías, que a su vez contienen indicadores cuya medición depende de microvariables o unidades operativas más básicas sobre las que se emiten juicios de valor, como se realizó con los instrumentos de recolección de información durante las visitas de campo a las empresas; en este sentido se obtuvo la siguiente matriz de evaluación y análisis:

\section{TABLA 4}

Matriz de operacionalización de variables para la medición de capacidades de innovación empresarial (IE).

\begin{tabular}{|c|c|c|c|}
\hline Variables & Subvariables & Indicadores & Microvariables medibles \\
\hline \multirow{13}{*}{ 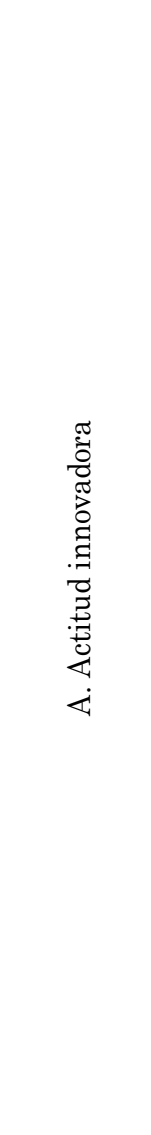 } & \multirow{8}{*}{$\begin{array}{l}\text { Promoción de } \\
\text { la cultura de la } \\
\text { innovación }\end{array}$} & \multirow{5}{*}{$\begin{array}{l}\text { Liderazgo de directivos } \\
\text { (propietarios-gerentes) } \\
\text { para el logro de } \\
\text { cambios innovadores }\end{array}$} & Conocimiento y experiencia de la IE de directivos \\
\hline & & & $\begin{array}{l}\text { Pensamiento creativo y generador de ideas de valor de } \\
\text { directivos }\end{array}$ \\
\hline & & & $\begin{array}{l}\text { Forma de asumir cambios empresariales por parte de } \\
\text { los directivos }\end{array}$ \\
\hline & & & $\begin{array}{l}\text { Existencia de responsable o equipo encargado de la } \\
\text { gestión de la IE }\end{array}$ \\
\hline & & & Disponibilidad de personal capacitado en IE \\
\hline & & \multirow{3}{*}{$\begin{array}{l}\text { Aptitud de } \\
\text { colaboradores } \\
\text { (trabajadores mandos } \\
\text { medios, operativos, } \\
\text { asesores) para la } \\
\text { innovación }\end{array}$} & $\begin{array}{l}\text { Existencia de programas, planes o acciones de } \\
\text { formación integral para la creatividad e IE }\end{array}$ \\
\hline & & & $\begin{array}{l}\text { Existencia de estrategia de incentivos a la generación } \\
\text { de ideas creativas }\end{array}$ \\
\hline & & & $\begin{array}{l}\text { Disponibilidad para la acción innovadora empresarial } \\
\text { de colaboradores }\end{array}$ \\
\hline & \multirow{5}{*}{$\begin{array}{l}\text { Recursos } \\
\text { internos } \\
\text { disponibles } \\
\text { para la } \\
\text { innovación }\end{array}$} & \multirow{3}{*}{$\begin{array}{l}\text { Sistemas de } \\
\text { información y } \\
\text { comunicación } \\
\text { para la innovación } \\
\text { empresarial }\end{array}$} & $\begin{array}{l}\text { Conocimiento y manejo de sistemas de información y } \\
\text { comunicación para la IE }\end{array}$ \\
\hline & & & Existencia de sistemas de información actualizados. \\
\hline & & & $\begin{array}{l}\text { Uso de los sistemas de información y comunicación } \\
\text { empresarial para tomar decisiones }\end{array}$ \\
\hline & & \multirow{2}{*}{$\begin{array}{l}\text { Disponibilidad de } \\
\text { recursos económicos } \\
\text { para la innovación }\end{array}$} & $\begin{array}{l}\text { Presupuesto asignado para la inversión en innovación } \\
\text { empresarial en último año }\end{array}$ \\
\hline & & & Presupuesto invertido en IE durante el último año \\
\hline
\end{tabular}




\begin{tabular}{|c|c|c|c|}
\hline Variables & Subvariables & Indicadores & Microvariables medibles \\
\hline \multirow{18}{*}{ 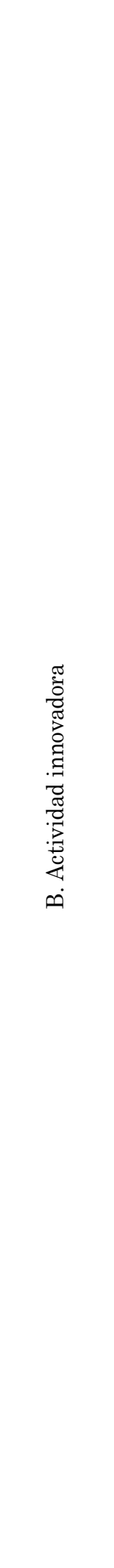 } & \multirow{6}{*}{$\begin{array}{l}\text { Innovación de } \\
\text { producto }\end{array}$} & $\begin{array}{l}\text { Conocimiento y } \\
\text { análisis del mercado }\end{array}$ & $\begin{array}{l}\text { Conocimiento y análisis de necesidades y expectativas } \\
\text { de clientes actuales y potenciales }\end{array}$ \\
\hline & & & Conocimiento y análisis de tendencias del mercado \\
\hline & & $\begin{array}{l}\text { Generación de ideas } \\
\text { creativas }\end{array}$ & $\begin{array}{l}\text { Existencia de proceso para promover la generación de } \\
\text { ideas creativas: responsables, procedimientos, recursos }\end{array}$ \\
\hline & & \multirow{2}{*}{$\begin{array}{l}\text { Diseño y desarrollo de } \\
\text { producto innovadores }\end{array}$} & $\begin{array}{l}\text { Diseño creativo e innovador de productos nuevos } \\
\text { o mejorados (especificaciones técnicas, deseos y } \\
\text { expectativas del producto) }\end{array}$ \\
\hline & & & $\begin{array}{l}\text { Programación del desarrollo del producto innovador } \\
\text { (incluye análisis de costos y articulación con otros } \\
\text { procesos empresariales.) }\end{array}$ \\
\hline & & $\begin{array}{l}\text { Evaluación de impacto } \\
\text { del producto innovador } \\
\text { en el mercado }\end{array}$ & $\begin{array}{l}\text { Evaluación de aceptación del producto (captación del } \\
\text { valor demandado por el cliente) }\end{array}$ \\
\hline & \multirow{6}{*}{$\begin{array}{l}\text { Innovación de } \\
\text { proceso }\end{array}$} & \multirow{3}{*}{$\begin{array}{l}\text { Incorporación de valor } \\
\text { a la cadena productiva } \\
\text { y distribuidora }\end{array}$} & $\begin{array}{l}\text { Investigación y conocimiento de nuevas tecnologías } \\
\text { existentes en el mercado: nuevas técnicas, } \\
\text { procedimientos, maquinaria o equipo especializado }\end{array}$ \\
\hline & & & $\begin{array}{l}\text { Aplicación de tecnología: incorporación de nuevas } \\
\text { técnicas, procedimientos, maquinaria o equipo } \\
\text { especializado }\end{array}$ \\
\hline & & & $\begin{array}{l}\text { Análisis de desempeño productivo de los procesos de } \\
\text { producción y distribución }\end{array}$ \\
\hline & & \multirow{3}{*}{$\begin{array}{l}\text { Mejora del desempeño } \\
\text { de procesos de } \\
\text { producción y } \\
\text { distribución }\end{array}$} & $\begin{array}{l}\text { Redefinición de procesos por adopción de nuevas } \\
\text { tecnologías y articulación con otros procesos. } \\
\text { (responsables, capacitación para implementación, } \\
\text { control y evaluación del impacto). }\end{array}$ \\
\hline & & & $\begin{array}{l}\text { Gestión de calidad, SYSO y gestión ambiental. } \\
\text { (control y mejora continua de procesos, seguridad y } \\
\text { salud ocupacional, impacto ambiental de la tecnología } \\
\text { adquirida) }\end{array}$ \\
\hline & & & $\begin{array}{l}\text { Análisis de costos de producción y distribución por } \\
\text { innovaciones (gestión de proveedores) }\end{array}$ \\
\hline & \multirow{4}{*}{$\begin{array}{l}\text { Innovación en } \\
\text { mercadeo }\end{array}$} & \multirow{2}{*}{$\begin{array}{l}\text { Satisfacción y conexión } \\
\text { con los clientes }\end{array}$} & Satisfacción del cliente \\
\hline & & & Estrategias de relacionamiento con el cliente \\
\hline & & $\begin{array}{l}\text { Nuevos métodos de } \\
\text { comercialización }\end{array}$ & Implementación y mejora de la gestión de mercadeo \\
\hline & & \multirow{3}{*}{$\begin{array}{l}\text { Nuevos métodos } \\
\text { organizativos }\end{array}$} & $\begin{array}{l}\text { Modelo de negocios basado en procesos de creación, } \\
\text { desarrollo y captación de valor e innovación }\end{array}$ \\
\hline & \multirow[t]{2}{*}{$\begin{array}{l}\text { Innovación de } \\
\text { organización }\end{array}$} & & $\begin{array}{l}\text { Estructura organizacional, relaciones y poder de toma } \\
\text { decisión para desarrollar el modelo generador de valor } \\
\text { y de innovación }\end{array}$ \\
\hline & & & $\begin{array}{l}\text { Proceso de gestión del conocimiento: Capacidad de } \\
\text { aprendizaje e intercambios de información y protección } \\
\text { del conocimiento. }\end{array}$ \\
\hline
\end{tabular}




\begin{tabular}{llll}
\hline Variables & Subvariables & \multicolumn{1}{c}{ Indicadores } & \multicolumn{1}{c}{ Microvariables medibles } \\
\hline & $\begin{array}{l}\text { Información } \\
\text { para la }\end{array}$ & $\begin{array}{l}\text { Fuentes y acceso a la } \\
\text { información }\end{array}$ & Gestión de la información \\
& $\begin{array}{l}\text { Conocimiento y acceso } \\
\text { innovación }\end{array}$ & Gestión de la tecnología empresarial \\
\cline { 2 - 4 } & $\begin{array}{l}\text { Relaciona- } \\
\text { miento con el } \\
\text { entorno para la la }\end{array}$ & $\begin{array}{l}\text { Estructura de } \\
\text { relacionamiento con el } \\
\text { entorno }\end{array}$ & Base de datos de agentes del sistema de innovación \\
& innovación & Redes o alianzas para la innovación \\
\hline
\end{tabular}

Fuente: Elaboración propia.

\section{Discusión}

La innovación empresarial es una herramienta de gestión empresarial fundamental en el contexto de competitividad vigente, cuyo reconocimiento, gestión y aplicación por parte de los empresarios, permite aumentar las capacidades empresariales de competitividad, especialmente en micro y pequeñas empresas cuyos modelos de gestión dependen de la planeación cortoplacista, para atender las necesidades individuales de autoempleo e informalidad.

Esta realidad empresarial de un gran número de pymes latinoamericanas caracterizadas por bajos niveles de formación de su capital humano, dificultad de acceso a recursos financieros externos, bajo índice de internacionalización y actividades que demandan baja tecnificación de sus procesos (Dini, Rovira y Stumpo, 2014, p. 10), realidad que no es ajena al contexto nortesantandereano en donde "El recurso humano en su gran mayoría está cualificado hasta el nivel técnico y en un bajo porcentaje es profesional o especializado" (Gonzáles, 2015, p. 18); lo que les obliga a un replanteamiento general para fortalecerse y aprovechar las oportunidades del mercado globalizado, que demanda creatividad e innovación para mantener la competitividad.
A su vez, la competitividad como capital intangible, exige un cambio de la filosofía directiva y una flexibilización suficiente para gestionar y desarrollar nuevas ideas tendientes a buscar, fidelizar y satisfacer constantemente a sus clientes con el fin de lograr un crecimiento empresarial sostenible.

En este sentido, la sistematización de las subvariables a partir de los referentes teóricos y la sistematización de datos obtenidos mediante las entrevistas a los actores empresariales del estudio, confirmaron las premisas antes desarrolladas y su corresponsabilidad con los escenarios de gestión y operación reconocidos en el trabajo de campo del equipo investigador.

Esta triangulación informativa permitió corroborar que la innovación empresarial solo es posible a partir de la cultura como un hábito organizacional y la formación continua implementada en la realidad empresarial, la cual parte en primera instancia, de una sensibilización y compromiso en todos los niveles gerenciales y operativos, la promoción de la gestión de ideas y la acción innovadora tendiente a cambiar la forma de realizar los procesos e implementar mejoras continuas guiadas por un modelo de gestión soportado en el que hacer innovador. En este sentido innovar se convierte en un hábito orga- 
nizacional, que debe estar soportado en la formación continua para la generación de cambios reales en los procesos y la mejora permanente.

Operativamente dichos cambios parten de inversiones graduales ejecutadas sobre estudios de necesidades, para motivar la generación de nuevas ideas que a su vez capitalicen experiencias acordes con la práctica real; el trabajo de campo permitió entender que la innovación empresarial no solo se representa con acciones materiales o grandes inversiones tecnológicas, sino que tiene su asidero en la formación integral; es decir, el conocimiento acumulado, así como la gestión de ideas creativas surgidas desde la experiencia empresarial, y la búsqueda constante de la satisfacción del mercado.

También pudo evidenciarse una reciente tendencia empresarial de la región, a investigar y reconocer tanto el entorno inmediato (regional - nacional) como el mundial, y con ello, articularse y gestionar capacidades con otras organizaciones e instituciones para sumar esfuerzos de beneficio recíproco y mejoramiento empresarial, para fortalecer el trabajo interno y aumentar el valor agregado de los productos y servicios a partir de la suma de esfuerzos colectivos.

Esto es especialmente entendido por los empresarios de la región, toda vez que conocen las dificultades de las micro y pequeñas empresas para afrontar los retos económicos y competitivos derivados de la globalización y la tecnología, solo son superables mediante la sumatoria de sus competencias y esfuerzos, especialmente ante la reciente coyuntura de cambios económicos regionales, y la posibilidad de apertura hacia nuevos mercados tras la atenuación de la actividad comercial con Venezuela.

\section{Conclusiones}

Se concluye que la capacidad de innovación empresarial implica una administración efectiva de los elementos claves de éxito; los internos como la actitud y las actividades innovadoras, y los externos, asociados con el relacionamiento entre organizaciones para mejorar la organización y la competitividad; dichos elementos, soportados en la gestión del conocimiento empresarial, la creatividad e innovación, permiten afrontarlos retos y cambios continuos del mercado, y fortalecen las ventajas competitivas para aprovechar las oportunidades del entorno.

La capacidad innovadora empresarial se fortalece con el liderazgo, los hábitos, la motivación y los incentivos de los actores de la empresa, lo cual fomenta cambios en el quehacer empresarial desde el modelo de gestión, la innovación y la asignación de recursos; la constante implementación y mejora de la cultura innovadora genera un valor creativo y diferenciador, tanto hacia el interior de la empresa, como en su relacionamiento con el entorno y sus productos.

El fortalecimiento cultural de la innovación empresarial debe adoptar un carácter endógeno y colaborativo desde la perspectiva empresarial, para lograr el desarrollo competitivo territorial mediante la alianza Universidad - Estado - Empresa (UEE), como entre promotor permanente de nuevo conocimiento a partir del intercambio de experiencias, y el apoyo a la gestión de proyectos innovadores de crecimiento empresarial regional.

La apuesta UEE resultante del diagnóstico de las 50 empresas nortesantandereanas investigadas, sugiere fortalecer las líneas de trabajo débiles para mejorar la capacidad innovadora empresarial, mediante acciones como: 1) fortalecer el pen- 
samiento creativo empresarial e incentivos; 2) diseñar e implementar la generación de ideas; 3) diseñar el programa de desarrollo de producto; 4) aplicar metodologías para evaluar la aceptación del producto; 5) vigilar nuevas tecnologías del mercado; 6) adoptar metodologías de análisis de desempeño productivo y costos de innovación; 8) activar las mejoras de gestión de mercadeo y del conocimiento; y 10) adelantar gestión con entidades de apoyo a la innovación en torno a la información y financiamiento.

\section{REFERENCIAS}

Ángel, B. (2005). La gestión de la innovación en las grandes empresas. Revista Lupa Empresarial. (11). [online]. Recuperado de http://www. ceipa.edu.co/lupa/index.php/lupa/article/view/107/208

Avendaño, C. (2012). Innovación: un proceso necesario para las pequeñas y medianas empresas del municipio de San José de Cúcuta, Norte de Santander. Semestre Económico, 15(31), 187-207.

Bes, T. y Kotler, P. (2011). Innovar para ganar. Barcelona: Urano.

CEPAL (2012). Políticas de innovación para pymes en América Latina. Santiago de Chile: Naciones Unidas.

Chiavenato, I. (2011). Planeación estratégica. México, D.F.: Mc Graw Hill.

Dini, M., Rovira, S. y Stumpo, G. (2014). Una promesa y un suspirar. Políticas de innovación para Pymes en América Latina. Santiago de Chile: Cepal.

Donate, M. y Guadamillas, F. (2010). Estrategia de gestión del conocimiento y actitud innovadora en empresas de castilla-la mancha. Un estudio exploratorio. Revista Investigaciones Europeas de Dirección y Economía de la Empresa, 16(1), 31-54.
Gobernación de Norte de Santander (2016). Plan de desarrollo para Norte de Santander 2016-2019. Recuperado de http://www.sednortedesantander. gov.co/sitio/images/documentos/informesdelsector/PDD\%20NDS\%20 2016-2019.pdf

González, J. (2015). Nivel de innovación y tecnología del sector manufacturero en Norte de Santander. Cuadernos Latinoamericanos de Administración, 11(20), 7-18.

Jaramillo, H., Lugones, G. \& Salazar, M. (2011). Normalización de Indicadores de Innovación Tecnológica en América Latina y el Caribe. Manual de Bogotá. Bogotá, D.C.: Colciencias/ OCYT. Recuperado de http://www. ricyt.org/manuales/doc_view/5-manual-de-bogota

Kotler, P., Armstrong, G. y Benassini, F.(2012). Fundamentos de Marketing. México, D.F.: Pearson.

OCDE, (2006). Manual de Oslo. Guía para la recogida e interpretación de datos de innovación. Recuperado de http://www.itq.edu.mx/convocatorias/manualdeoslo.pdf

Osterwalder, A. y Pigneur, Y. (2011). Generación modelos de negocio. Madrid: Libros PAPF.

Pérez, M., Hernández, J., Acosta, I., Chumaceiro, A. (2009). Consideraciones teóricas para el análisis de las Pequeñas y Medianas Empresas como fuente de generación de empleo y su correspondencia ética con la Sociedad. Revista Formación Gerencial, 8(2). 272-297.

Porter, M. (2001). La ventaja competitiva de las naciones. Harvard Business Reviw, 85(11). 69-95. 


\section{Biodata}

Claudia Milena Pérez es Ingeniera industrial de la Universidad Libre (Cúcuta, Colombia) con Mg.S. en Dirección del desarrollo local de la Universidad Francisco de Paula Santander (Colombia) - Instituto Superior de Educación, Administración y Desarrollo-ISEAD (Madrid, España) y Especialista en Aseguramiento de la Calidad de la Universidad Francisco de Paula Santander (Colombia). Integrante del grupo GIDTI, Coordinadora Semillero Reinnova y Docente investigadora de pregrado de la Corporación Universitaria Minuto de Dios, Centro Regional Cúcuta (Colombia). https:// orcid.org/0000-0002-3705-4673 